\title{
The case study: should it be dismissed as anecdotal and valueless, or be retained as a time-honored method of bedside teaching?
}

\author{
Richard Kones ${ }^{* 1,2}$ \\ ${ }^{1}$ Cardiometabolic Research Institute, Texas Medical Center, Houston, USA \\ ${ }^{2}$ Editor-in-chief for Cardiovascular Disorders and Medicine
}

In this modern era of randomized controlled trials (RCT) seeking tens of thousands of participants, most clinicians wonder about the value of case studies. Is there any relevance at all in devoting precious time in producing, publishing, and reading them, when competitive activities may be more rewarding in one way or another? In 2001, McWhinney considered this question, and a few of the points made are worth revisiting [1]. The first is that the learning from a case study is different from several tables, data collections, or survival curves. While the latter are valuable and necessary for guideline-writers and academics, there is a unique context to each case that affects practitioners differently. A case touches clinicians personally, in that multiple simultaneous reactions are evoked. Our entire knowledge base and experience is directed to the individual patient, rather than be confined to the more abstract ideas found in other learning vehicles. We are immediately challenged, but also confronted by the human element involved in patient care. As the narrative develops, we match our own ability to render a differential diagnosis and consider what we would do, as compared with what was done by the narrators. Can he/ she arrive at a better treatment plan more efficiently? Further, when differences occur, the thoughtful physician always wants to know why, primarily to improve his/her skills. In some respects, one could argue that the case study or presentation brings a different qualitative experience to the physician, as compared with reading a detailed systematic review or RCT. The second point made is that doctors treat patients, either at the bedside or in the office examining setting, and that model is still retained in making "rounds" on hospital floors when teaching students. One might liken the case study to a form of clinicopathological conference (CPC), once a basic (and well-attended) tradition in medical school affiliated hospitals. These concepts have not been rejected but have taken different forms over time: (i) patients are still treated individually, with modifications according to more rigid guidelines, (ii) additional room for incorporation of patients' views exists in shared decision-making discussions, and indeed, (iii) fundamentals of critical and clinical decision skills are assessed in interactive teaching tools. Interactive case studies are typically found in short learning skits and quizzes on medical news sites, in CME evaluations, and in evaluation of diagnostic and therapeutic prowess in medical licensing and board examinations. Obviously, medicine is now in a different era, and over-reliance upon individual cases must be tempered with restricted generalizability and other limitations. Therefore it is important to present a differential, sufficient perspective, and some discussion in case reports to forestall premature conclusions, faulty reasoning, and "anchoring bias." However, for most readers there is likely something to learn in every clinical case.
In this second volume of the Open Access Text Cardiovascular Disorders and Medicine (CDM) Journal, Bolognesi writes about am ECG with a delta wave, short $\mathrm{PR}$ interval and changes mimicking an inferior wall infarction in a 19-year-old male athlete. This paper highlights the current debate about how to properly screen healthy athletes, and appropriately investigate those with symptoms or signs of heart disease. Sudden cardiac death of athletes is a tragedy from every standpoint, but benefits of screening are debated. When there is an abnormality on the ECG, what procedures are considered sufficient for the consultant to "clear" the patient for full activity-with confidence, and why? Even early repolarization, once considered a "normal" variant, is no longer considered benign. Do you agree with the authors' decision?

Beam and Prahlow report on a 47-year-old African-American man with untreated hypertension and first-onset seizure. In the emergency department, his glucose was $173 \mathrm{mg} / \mathrm{dL}$, and neither clinical nor ECG/EEG findings showed sufficient reason for admission. After $3 \mathrm{~h}$ without any seizure activity, he was discharged, only to return with a terminal ventricular arrhythmia. On autopsy, there was diffuse, severe atherosclerosis and hypertensive heart disease.

Caiati et al. report about a 52-year-old black woman from Italy with diabetes type 2, the metabolic syndrome, hypertension, a history of significant coronary sclerosis on coronary angiography 2 years earlier, and chest pain. The patient exhibited dyskinesia and improved; she was discharged and hours later experienced severe pain, syncope, and cardiac arrest. Pathological examination showed mild left ventricular hypertrophy and non-occlusive coronary sclerosis, histology consistent with recent acute ischemia, and immunohistochemistry showing perineural infiltration of cardiac autonomic fibers. Hence, the events were attributed to an intense coronary vasospastic response superimposed upon preexisting disease. Variant or Prinzmetal's angina, of course, may typically be severe, occur at rest, at night especially the early morning hours, and is multifactorial, being associated with genetic loading, tobacco or cocaine use, exposure to cold weather, hyperventilation, nitric oxide deficiency, inflammation and oxidative

Correspondence to: Richard Kones, Medical Director, Cardiometabolic Research Institute, 8181 Fannin St, Unit 314, Houston, TX 77054, USA, Editor-in-Chief for Cardiovascular Disorders and Medicine, E-mail: medkones@gmail.com

Received: January 03, 2017; Accepted: January 18, 2017; Published: January 21, 2017 
stress, monocytosis, C-reactive protein levels, stress, and autonomic nervous dysfunction. In the majority of instances, however, there is no readily identifiable cause, and one must be content with achieving relief.

In a mini-review, Heuser then treats us to a cogent discussion of adequate support when placing balloons or stents. He relates his experience using a variant of the anchoring balloon technique in coronary and other complex interventions, without which stents could not be placed, otherwise leaving the patient unprotected. There are some excellent illustrations in this "how to" discussion of crossing chronic total occlusion or complex lesions with enhanced support. For readers, whose appetites are piqued for more, the author provides some recent references.

Last, Kiuchi and Chen studied the incidence of bradyarrhythmias in a hypertensive population with chronic kidney disease (CKD) patients according to the degree of obstructive sleep apnea (OSA). Bradyarrhythmias associated with OSA are more common than generally appreciated, may occur in multiple severe episodes, chiefly nocturnal, and include frequent pauses due either to sinus arrest or complete heart block, even $\geq 13$ s duration [2]. Ventricular asystole may occur in $7.5 \%$ of all patients with OSA, but when OSA is severe, the frequency can rise to $20 \%$ of patients. These numbers are a wake-up call, since a substantial number of individuals with OSA remain undiagnosed, yet treatment is highly effective in lowering cardiovascular morbidity and mortality [3].

Both anatomic and electrical remodeling result from multifactorial factors in these disorders: hypoxia, hypercapnia, inflammation, oxidative stress, autonomic changes, acute atrial stretch, negative intrathoracic pressure, higher intravascular volume, and left ventricular diastolic dysfunction. Increased peripheral sympathetic activity is a potent risk factor for cardiovascular events and CKD $[3,4]$.

\section{References}

1. McWhinney IR (2001) The value of case studies. European Journal of General Practice 7: 3, 88-89.

2. Simantirakis EN, Schiza SI, Marketou ME, Chrysostomakis SI, Chlouverakis GI, et al. (2004) Severe bradyarrhythmias in patients with sleep apnoea: the effect of continuous positive airway pressure treatment: a long-term evaluation using an insertable loop recorder. Eur Heart J 25: 1070-1076. [Crossref]

3. Hersi AS (2010) Obstructive sleep apnea and cardiac arrhythmias. Ann Thorac Med 5 10-17. [Crossref]

4. Filgueiras-Rama D, Arias MA, Iniesta A, Armada E, Merino JL, et al. (2013) Atrial arrhythmias in obstructive sleep apnea: underlying mechanisms and implications in the clinical setting. Pulm Med: 426758. [Crossref]

Copyright: (C2017 Kones R. This is an open-access article distributed under the terms of the Creative Commons Attribution License, which permits unrestricted use, distribution, and reproduction in any medium, provided the original author and source are credited. 